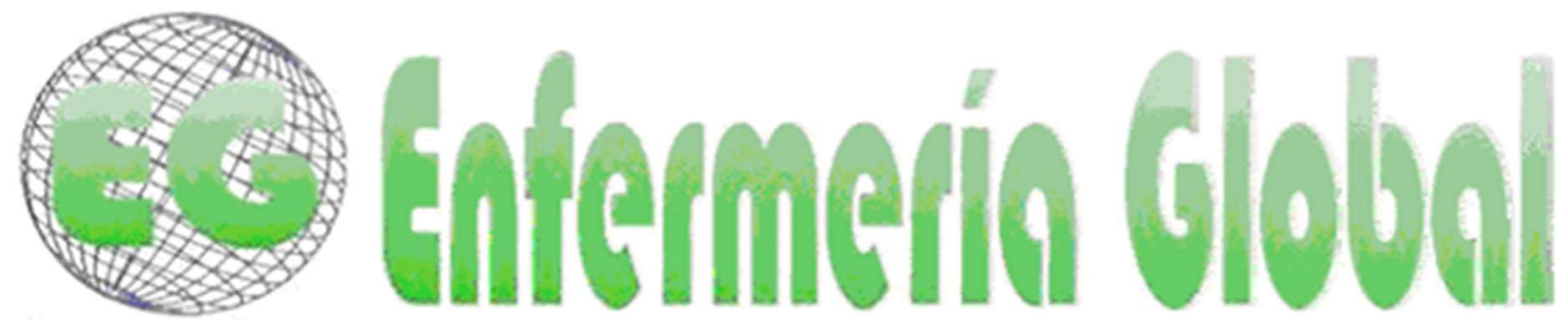

\title{
ORIGINALES
}

\section{Mejora de la calidad de vida en cuidadores informales de personas dependientes mediante talleres educacionales}

Improvement of quality of life for informal caregivers of dependent persons through educational workshops

\section{María Dolores Guijarro-Requena ${ }^{1}$ \\ María Nieves Marín-Campaña ${ }^{1}$ \\ María Isabel Pulido-Lozano ${ }^{1}$ \\ Rocío Melka Romero-Carmona ${ }^{1}$ \\ Luis Gabriel Luque-Romero²}

\begin{abstract}
1 Unidad de Gestión Clínica de Rehabilitación Sevilla. Distrito Sanitario Aljarafe-Sevilla Norte. Sevilla, España. nieves.marin.sspa@juntadeandalucia.es

${ }^{2}$ Unidad de Investigación. Distrito Sanitario Aljarafe-Sevilla Norte. Facultad de Medicina. Departamento de Citología e Histología Normal y Patológica. Universidad de Sevilla. Sevilla, España.
\end{abstract}

\section{https://doi.org/10.6018/eglobal.478351}

Recibido: $27 / 04 / 2021$

Aceptado: $21 / 09 / 2021$

\section{RESUMEN:}

Introducción: El envejecimiento de la población está asociado a un incremento de personas dependientes, estimándose que el $15 \%$ vive con alguna discapacidad. La prestación de cuidados a familiares supone una responsabilidad añadida asociada a problemas relacionados con el cuidado, que pueden repercutir negativamente en los cuidadores. Por ello, planteamos diseñar y evaluar un programa de cuidados, basado en la educación de los cuidadores, midiendo los conocimientos, la percepción del dolor, la carga emocional y la calidad de vida relacionada con la salud.

Objetivo: El objetivo principal es mejorar la calidad de vida de los cuidadores informales mediante intervenciones formativas, con el fin de disminuir la incapacidad causada por las tareas del cuidado.

Método: Participaron 99 cuidadores pertenecientes al Distrito Sanitario Aljarafe-Sevilla Norte de Atención Primaria, divididos en grupo control e intervención. Diseñamos dos talleres educacionales sobre programas de atención a cuidadores informales. Cumplimentaron un cuestionario de conocimientos, y diferentes escalas validadas relacionadas con el dolor, los estados de salud y la calidad de vida.

Resultados: La mayor parte de los cuidadores presentaban problemas de espalda tomando medicación para ello. La intervención implementada se asoció a un descenso del dolor durante actividades básicas de la vida diaria, los cuidados y el descanso, y disminución del índice de esfuerzo en ambos grupos.

Conclusiones: Los programas de intervención en cuidadores informales de pacientes dependientes resultan eficaces para mejorar el estado de salud y la calidad de vida de este colectivo por lo que se debe fomentar su realización desde la Atención Primaria. 
Palabras clave: Cuidadores, evaluación del dolor, educación en salud, índice de esfuerzo del cuidador, calidad de vida.

\begin{abstract}
:
Introduction: The ageing of the population is associated with an increase in the number of dependent people, with an estimate of $15 \%$ living with a disability. The provision of care to family members entails an added responsibility associated with care-related problems, which can have a negative impact on caregivers. Therefore, we propose to design and evaluate a caregiving programme based on caregiver education, measuring knowledge, pain perception, emotional burden and health-related quality of life.

Objective: The main objective is to improve the quality of life of informal caregivers through training interventions, in order to decrease the disability caused by caregiving tasks.

Method: 99 caregivers belonging to the Aljarafe-Northern Seville Primary Care Health District participated, divided into control and intervention groups. We designed two educational workshops on care programmes for informal caregivers. They completed a knowledge questionnaire, and different validated scales related to pain, health states and quality of life.

Results: Most of the caregivers presented back problems, for which they received pharmaceutical treatment. The implemented intervention was associated with a decrease in pain during basic activities of daily living, care and rest, as well as a reduction in strain index in both groups.

Conclusions: Intervention programmes for informal carers of dependent patients are effective in improving the state of health and quality of life of this group, and their implementation should therefore be encouraged in primary care.
\end{abstract}

Keywords: Caregivers, pain evaluation, health education, caregiver effort index, quality of life.

\title{
INTRODUCCIÓN
}

El envejecimiento de la población está asociado a un incremento de las personas dependientes $^{(1)}$, llegándose a estimar que alrededor del $15 \%$ de la población mundial vive con algún tipo de discapacidad. Una persona dependiente se define como aquella que necesita de la ayuda de otra persona para realizar sus actividades básicas de la vida diaria. La mayor parte de estos cuidados, entre los que podemos destacar la alimentación, la vestimenta y las transferencias; son asumidas por los familiares de la persona dependiente. De esta manera, la familia se ha convertido en el principal proveedor de los mismos, llegando a establecerse una red de cuidadores informales $y / 0$ familiares, que garantizan estas labores de una forma no remunerada $^{(2)}$. Si la esperanza de vida sigue aumentando y la población sigue envejeciendo, se nos plantea el problema de la disponibilidad de cuidadores informales y la necesidad de realizar importantes reformas de los Sistemas Sanitarios público/privados y de la Atención Social ${ }^{(3,4)}$.

La prestación de cuidados a un familiar supone una responsabilidad añadida. En muchas ocasiones, los cuidadores informales tienen que renunciar a su trabajo y reducir el número de actividades sociales para poder garantizar una atención adecuada a la persona dependiente. Esta nueva responsabilidad se ha asociado a problemas tales como la sobrecarga de roles y la falta de información, junto con problemas económicos y cambios en el estado de salud del cuidador. Todo ello conlleva una disminución de la calidad de vida(5) y la aparición del denominado "síndrome del cuidador"(6). Con mucha frecuencia nos encontramos que el dolor de espalda es un síntoma asociado a esta actividad de cuidado. Según Zuluaga y


límite de acción e incluso superior al valor de rotura de la unidad disco-vertebral. Las lesiones a nivel físico (lesiones de espalda, problemas musculares y problemas osteoarticulares) suponen un enorme gasto económico y social, por el elevado 
número de bajas laborales y el desgaste emocional que conlleva para las personas cuidadoras $^{(5)}$.

Aunque la calidad de vida, la carga de la enfermedad, la insatisfacción, el estrés, y la depresión de los cuidadores informales se han estudiado extensamente ${ }^{(8)}$, y algunos estudios han abordado el dolor lumbar en cuidadores formales ${ }^{(9)}$, solo determinados estudios abordan el dolor lumbar de dichos cuidadores en el domicilio(8,10). Por todo ello, nos hemos planteado diseñar y evaluar la eficacia de una actividad formativa dirigida a los cuidadores familiares que aborden temas sobre cinesiterapia, ergonomía y técnicas de relajación. Además, en esta actividad formativa se han impartido contenidos relacionados con el manejo de la persona dependiente, con el fin de disminuir la incapacidad causada por el rol del cuidador. Para determinar la eficacia de la actividad vamos a medir la variación entre la situación basal y la situación final de la percepción de dolor, de la carga emocional y de la calidad de vida de estos cuidadores informales.

\section{MATERIAL Y MÉTODOS}

Se ha realizado un estudio multicéntrico cuasiexperimental con un grupo control sobre una muestra de cuidadores atendidos por un equipo de fisioterapeutas del Distrito Sanitario Aljarafe-Sevilla Norte en el ámbito de la Atención Primaria, entre el periodo comprendido entre el año 2015 y el año 2019.

La muestra de nuestro estudio se seleccionó mediante una lista de números aleatorios, entre los cuidadores informales que constaban en los listados de grandes dependientes de nuestro Distrito. Se consideraron como candidatos para participar en el estudio a aquellos cuidadores que tenían a su cargo a una persona dependiente con un índice de Barthel inferior a 60 puntos y que estuviera encamada total o parcialmente. Además, otro requisito para participar en el estudio ha sido que los cuidadores tenían que haber dado su consentimiento de forma explícita. Se excluyeron de nuestro estudio a aquellos cuidadores con patologías muy limitantes o agudas y a aquellos que tuviesen una edad inferior a 18 años. Se calculó un tamaño muestral de 100 cuidadores informales, distribuidos por mitad en cada grupo de estudio para tener un nivel de confianza del $95 \%$ y una potencia del $80 \%$.

Se diseñó una actividad formativa que consistió en la realización de dos talleres educacionales basados en la mejor evidencia científica disponible sobre programas de educación en el manejo del familiar dependiente y en higiene postural (taller 1), estiramientos, y técnicas de relajación (taller 2). El contenido del taller teórico-práctico fue consensuado por todos los investigadores participantes en el estudio y la documentación se entregó a los cuidadores al final del segundo taller.

Antes del inicio (pre) y al final (post) de la actividad formativa se evaluaron los conocimientos de los participantes, mediante un cuestionario autocumplimentado diseñado ad-hoc validado por expertos. Este cuestionario constaba de 10 preguntas sobre conocimientos de ergonomía, cambios posturales y transferencias; así como características sociodemográficas del cuidador (anexo 1). Igualmente, se pasaron las siguientes escalas validadas: la escala numérica visual de dolor $(E N V)^{(11)}$, el pictograma de localización de puntos dolorosos (figura 2)(12,13), el Cuestionario del Dolor en español ${ }^{(12,13)}$, el Índice de esfuerzo del Cuidador $\left(\right.$ IEC) ${ }^{14}$ y la escala de 
calidad de vida $(E Q-5 D)^{(15,16)}$. De forma complementaria, se les preguntó sobre la toma de analgésicos.

Una vez que se recogió toda la información basal, se les impartió la actividad formativa. Al finalizar dicha actividad, los cuidadores respondieron de nuevo al cuestionario de conocimientos y se evaluaron todas las habilidades y destrezas adquiridas en estos talleres.

Para conocer a lo largo del estudio el grado de cumplimiento del programa de ejercicios de estiramientos y de relajación se usó el test de adherencia de MoriskiGreen (MG) ${ }^{(17,18)}$ cambiando el término "tratamiento" por el de "ejercicios recomendados". El cronograma de cuestionarios realizados puede verse en la tabla 1.

Tabla 1. Cronograma de cuestionarios.

\begin{tabular}{|c|c|c|c|c|}
\hline Test & Basal & 3 meses & 9 meses & 18 meses \\
\hline Cuestionario de conocimientos & $\nabla$ & $\nabla$ & & \\
\hline ENV dolor & $\nabla$ & & & $\nabla$ \\
\hline Pictograma de dolor & $\nabla$ & & & $\nabla$ \\
\hline Cuestionario español del dolor & $\nabla$ & & & \\
\hline Índice de esfuerzo del cuidador & $\nabla$ & & & $\nabla$ \\
\hline$E Q-5 D$ & $\nabla$ & & & $\nabla$ \\
\hline Cumplimiento terapéutico & $\nabla$ & $\nabla$ & $\nabla$ & $\nabla$ \\
\hline
\end{tabular}

ENV: escala numérica visual; EQ-5D: cuestionario EuroQoL de calidad de vida

Las variables resultado estudiadas fueron: la intensidad del dolor (ENV), la presencia de dolor asociado a las actividades básicas de la vida diaria, a las tareas de cuidados y al descanso, la calidad de vida (EQ-5D), el índice de esfuerzo del cuidador, y el nivel de conocimientos antes y después del taller. Las variables explicativas contempladas en el estudio fueron: la edad, el sexo y la ocupación de los cuidadores familiares; y el índice de Barthel de la persona dependiente.

Las variables cuantitativas se expresaron mediante medias y desviaciones estándar o mediana y recorrido intercuartílico dependiendo de su distribución. Las variables cualitativas se expresaron en número y porcentaje. Se realizó un análisis inferencial utilizando la prueba de chi cuadrado para variables cualitativas. Para las cuantitativas se utilizó la t de Student en aquellas con distribución normal y el test de $U$ de MannWhitney para las variables que no seguían una distribución normal. Finalmente, se realizó un análisis de regresión logística binaria tomando como variable resultado el Índice de esfuerzo del cuidador al final del estudio recodificado en bajo/alto (alto: IEC $>7$ puntos). Todos los análisis se diseñaron bilaterales, aceptándose un nivel de confianza de 95\%. El análisis se realizó mediante IBM-SPSS v.22.

El estudio fue aprobado por el comité de ética de investigación de referencia de nuestra zona, en concreto el Hospital Universitario Virgen Macarena y el Hospital Regional Universitario Virgen del Rocío. 


\section{RESULTADOS}

Se analizaron los cuestionarios aportados por 99 cuidadores informales, de las que 91 $(91.9 \%)$ eran mujeres. La edad media (DS) fue de 53.8 (8.1) (intervalo de confianza del 95\%, IC95\%: 52.1-55.4) años. Del total, 46 (46.5\%) participaron en el grupo de intervención. A lo largo del estudio, $20(20.2 \%)$ fueron censurados, $8(8.1 \%)$ por abandono y 12 (12.1\%) por fallecimiento de la persona dependiente (figura 1).

\section{Figura 1: Flujograma de pacientes}

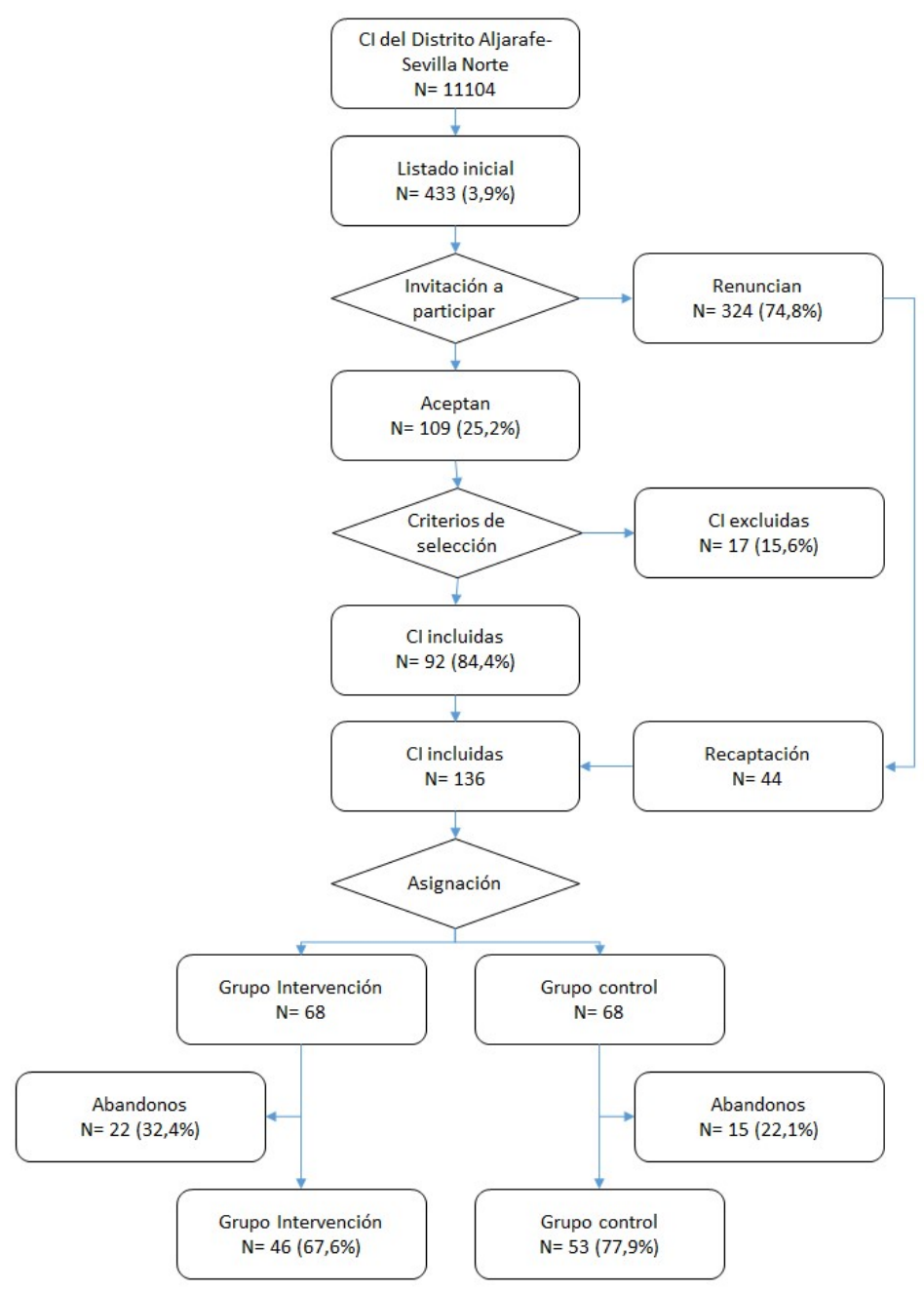

El índice de Barthel medio (DS) de las personas dependientes fue de 25.5 (19.8) (IC95\%: 20.0-30.9) puntos. No se encontraron diferencias con significación estadística entre el grupo control y el grupo de intervención en las variables medidas en el momento basal (tabla 2). 
Tabla 2: Distribución de las variables por grupo de estudio en el momento basal

\begin{tabular}{|c|c|c|c|c|}
\hline Variable & $\begin{array}{l}\text { Global } \\
N=99\end{array}$ & $\begin{array}{l}\text { Intervención } \\
46(46,5 \%)\end{array}$ & $\begin{array}{l}\text { Control } \\
53(53,5 \%)\end{array}$ & valor $p$ \\
\hline Edad, $\square \pm$ DS (IC 95\%) & $\begin{array}{c}53,8 \pm 8,1(52,1- \\
55,4)\end{array}$ & $\begin{array}{c}52,9 \pm 7,4(50,7- \\
55,1)\end{array}$ & $\begin{array}{c}54,5 \pm 8,7(52,1- \\
56,9)\end{array}$ & 0.331 \\
\hline \multicolumn{5}{|l|}{ Sexo, N (\%) } \\
\hline Mujer & $91(91,9)$ & $42(91,3)$ & $49(92,5)$ & 0.834 \\
\hline Hombre & $8(8,1)$ & $4(8,7)$ & $4(7.5)$ & \\
\hline $\begin{array}{l}\text { Índice de Barthel }{ }^{*}, \square \pm \\
\text { DS (IC 95\%) }\end{array}$ & $\begin{array}{c}25,5 \pm 19,8(20,0- \\
30,9)\end{array}$ & $\begin{array}{c}24,4 \pm 21,3(15,6- \\
33,2)\end{array}$ & $\begin{array}{c}26,4 \pm 18,6(19,2- \\
33,6)\end{array}$ & 0.591 \\
\hline Censurados, N (\%) & $20(20,2)$ & $8(17,4)$ & $12(22,6)$ & 0.516 \\
\hline \multicolumn{5}{|l|}{$\begin{array}{l}\text { Antecedentes } \\
\text { osteomusculares, N (\%) }\end{array}$} \\
\hline Artrosis & $18(18,2)$ & $8(17,4)$ & $10(18,9)$ & 0.849 \\
\hline Fibromialgia & $7(7,1)$ & $4(8,7)$ & $3(5,7)$ & 0.557 \\
\hline Osteoporosis & $6(6,1)$ & $2(4,3)$ & $4(7,5)$ & 0.506 \\
\hline Patología del hombro & $2(2,0)$ & $2(4,3)$ & $0(0,0)$ & 0.213 \\
\hline Raquialgia & $35(35,4)$ & $15(32,6)$ & $20(37,7)$ & 0.595 \\
\hline $\begin{array}{l}\text { Dolor durante las tareas } \\
\text { cuidador, } \mathrm{N}(\%)\end{array}$ & $53(53,5)$ & $25(54,3)$ & $28(52,8)$ & 0.88 \\
\hline $\begin{array}{l}\text { Dolor durante el } \\
\text { descanso, } \mathrm{N}(\%)\end{array}$ & $48(48,5)$ & $20(43,5)$ & $28(52,8)$ & 0.353 \\
\hline $\mathrm{EQ}-5 \mathrm{D}, \square \pm \mathrm{DS}$ (IC 95\%) & $65 \pm 21,3(60,7-69,2)$ & $\begin{array}{c}65,5 \pm 20,5(59,4- \\
71,6) \\
\end{array}$ & $\begin{array}{c}64,5 \pm 22,3(58,4- \\
70,7) \\
\end{array}$ & 0.791 \\
\hline $\begin{array}{l}\text { ENV Dolor, } \square \pm \text { DS (IC } \\
95 \%)\end{array}$ & $5,0 \pm 2,4(4,5-5,4)$ & $4,9 \pm 2,3(4,2-5,6)$ & $5,0 \pm 2,4(4,3-5,7)$ & 0.736 \\
\hline IEC, $\square \pm$ DS (IC 95\%) & $7,7 \pm 3,0(7,1-8,3)$ & $8,0 \pm 2,6(7,2-8,8)$ & $7,4 \pm 3,3(6,5-8,3)$ & 0.427 \\
\hline
\end{tabular}

En relación al parentesco el $65.4 \%$ de los cuidadores eran hijas/os, el $13.5 \%$ nueras o yernos. Con respecto al estado civil, el $88 \%$ estaban casados y la profesión más frecuente fue la de ama de casa en un $64,0 \%$, la agrícola en un $20,0 \%$ y otras con un $16 \%$.

Del total de cuidadores, $75(77.3 \%)$ referían tener problemas de espalda; el resto describían distintos problemas osteomusculares. En el test de localización de puntos dolorosos (pictograma), el 75.6\% de los cuidadores del grupo de intervención situaron como zona de dolor más frecuente la región cervical, mientras que en el grupo control la situaron en la región lumbo-sacra (70.2\%) (figura 2). 
Figura 2. Pictograma que representa la distribución de puntos dolorosos por grupo de estudio

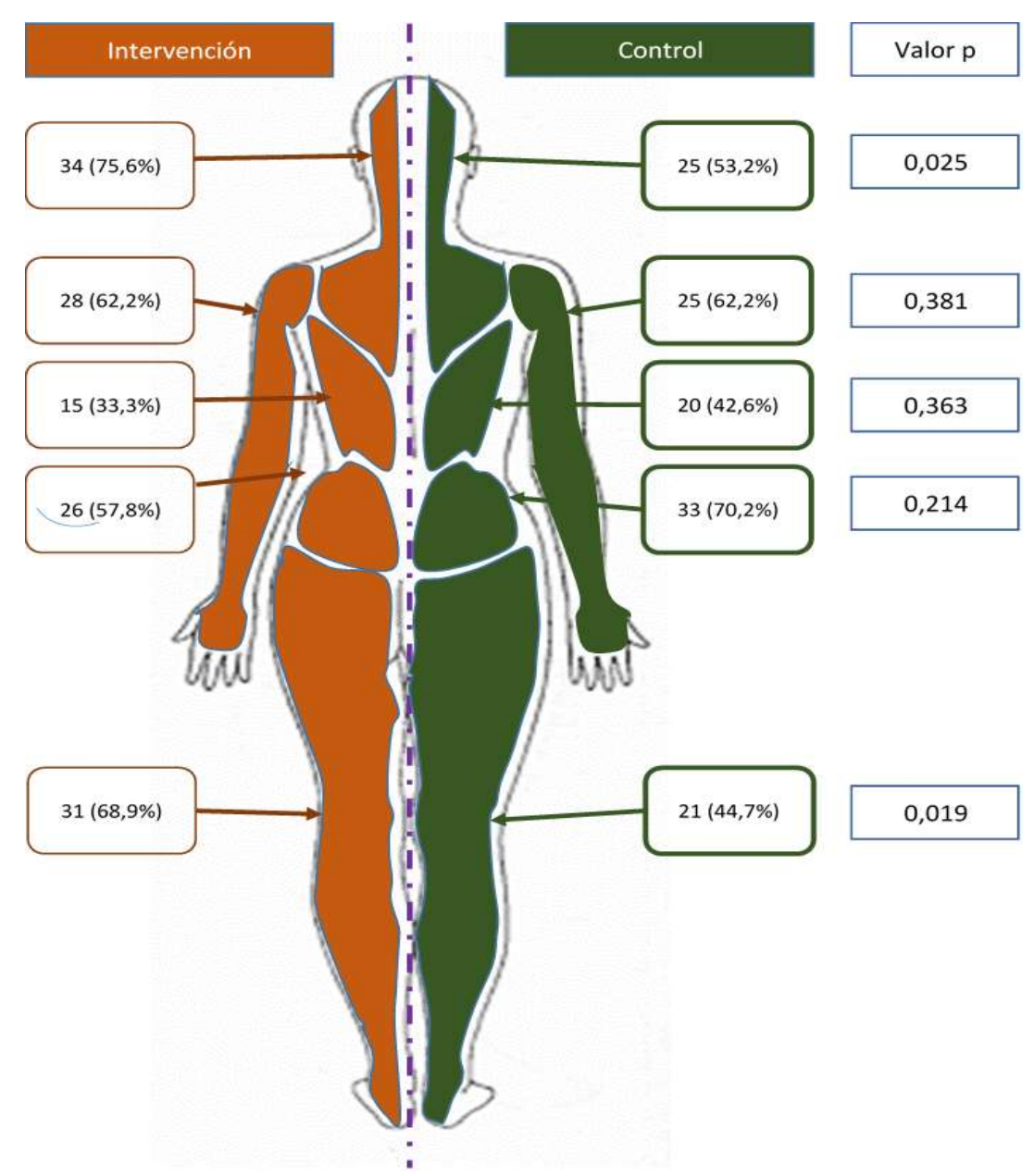

Solo $62(82.7 \%)$ tenían registrado el diagnóstico clínico en su historia clínica de salud y $64(85.3 \%)$ tenían prescrita medicación para este tipo de problemas. Sólo uno de ellos se automedicaba. A lo largo del estudio la frecuencia de cumplidores se mantuvo estable entre el $54 \%-56 \%$ (tabla 3 ).

Tabla 3. Test de adherencia al programa de entrenamiento impartido en la población de intervención (modificado a partir del test de Moriski-Green).

\begin{tabular}{llll}
\hline Test de adherencia terapéutica & $\mathbf{3}$ meses & $\mathbf{9}$ meses & $\mathbf{1 8}$ meses \\
\hline Total de respondedores al test, $\mathbf{N}(\%)$ & $36(78,3)$ & $36(78,3)$ & $35(76,1)$ \\
\hline $\begin{array}{c}\text { ¿Olvida alguna vez los ejercicios enseñados y las } \\
\text { indicaciones recibidas? }\end{array}$ & $20(43,5)$ & $20(43,5)$ & $19(41,3)$ \\
\hline $\begin{array}{l}\text { ¿Realiza las indicaciones aprendidas a las horas } \\
\text { indicadas? }\end{array}$ & $21(45,7)$ & $23(50,0)$ & $23(50,0)$ \\
\hline $\begin{array}{c}\text { Cuando se encuentra bien, ¿ deja de realizar los } \\
\text { ejercicios? }\end{array}$ & $15(32,6)$ & $24(52,2)$ & $18(39,1)$ \\
$\begin{array}{l}\text { ¿Aunque crea que le sientan mal los ejercicios } \\
\text { continua haciéndolos? }\end{array}$ & $21(45,7)$ & $23(50)$ & $19(41,3)$ \\
\hline Incumplimiento, N (\%) & & & \\
\hline$\quad$ Cumplidores & $10(21,7)$ & $10(21,7)$ & $10(21,7)$ \\
\hline Incumplidores & $26(56,5)$ & $26(56,5)$ & $25(54,3)$ \\
\hline
\end{tabular}


En relación a la calidad de vida de los cuidadores informales, esta intervención ha mejorado las puntuaciones en las subescalas del cuestionario EQ-5D: cuidado personal y actividades cotidianas de manera estadísticamente significativa en el grupo de intervención en relación al grupo control $(p=0.0005$ y $p=0.001)$, respectivamente. En la subescala de dolor malestar no se alcanzó la significación estadística, pero si se aprecia una menor proporción de personas con dolor en el grupo de intervención que en el grupo control $(p=0.082)$ (tabla4).

Tabla 4. Calidad de vida al inicio y al final del estudio según el grupo de estudio.

\begin{tabular}{|c|c|c|c|c|c|c|}
\hline \multirow[b]{2}{*}{ Subescalas EQ-5D, N (\%) } & \multicolumn{3}{|c|}{ Intervención } & \multicolumn{3}{|c|}{ Control } \\
\hline & Basal & $\begin{array}{c}18 \\
\text { meses }\end{array}$ & valor $p$ & Basal & $\begin{array}{c}18 \\
\text { meses }\end{array}$ & $\begin{array}{c}\text { valor } \\
p\end{array}$ \\
\hline $\begin{array}{l}\text { a) Movilidad } \\
\text { No tengo problemas } \\
\text { Tengo algunos problemas }\end{array}$ & $\begin{array}{c}26 \\
(72.2) \\
10 \\
(27.8)\end{array}$ & $\begin{array}{l}25(69.4) \\
11(30.6)\end{array}$ & 1.000 & $\begin{array}{c}34 \\
(82.9) \\
7(17.1)\end{array}$ & $\begin{array}{c}32(78.0) \\
9(22.0)\end{array}$ & 0.687 \\
\hline $\begin{array}{l}\text { b) Cuidado personal } \\
\text { No tengo problemas } \\
\text { Tengo algunos problemas } \\
\text { Soy incapaz }\end{array}$ & $\begin{array}{c}32 \\
(88.9) \\
4(11.1) \\
0(0.0)\end{array}$ & $\begin{array}{c}30(83.3) \\
5(13.9) \\
1(2.8) \\
\end{array}$ & 0.0005 & $\begin{array}{c}38 \\
(92.7) \\
3(7.3) \\
0(0.0)\end{array}$ & $\begin{array}{c}40(97.6) \\
1(2.4) \\
0(0.0) \\
\end{array}$ & 0.073 \\
\hline $\begin{array}{l}\text { c) Actividades cotidianas } \\
\text { No tengo problemas } \\
\text { Tengo algunos problemas } \\
\text { Soy incapaz }\end{array}$ & $\begin{array}{c}24 \\
(66.7) \\
11 \\
(30.6) \\
1(2.8) \\
\end{array}$ & $\begin{array}{c}21(58.3) \\
14(38.9) \\
1(2.8) \\
\end{array}$ & 0.001 & $\begin{array}{c}26 \\
(65.0) \\
14 \\
(35.0) \\
0(0.0) \\
\end{array}$ & $\begin{array}{c}22(55.0) \\
18(45.0) \\
0(0.0) \\
\end{array}$ & 0.101 \\
\hline $\begin{array}{l}\text { d) Dolor / Malestar } \\
\text { No tengo problemas } \\
\text { Tengo moderado dolor/malestar } \\
\text { Tengo mucho dolor/malestar }\end{array}$ & $\begin{array}{c}10 \\
(28.6) \\
19 \\
(54.3) \\
6(17.1) \\
\end{array}$ & $\begin{array}{c}5(14.3) \\
24(68.6) \\
6(17.1) \\
\end{array}$ & 0.082 & $\begin{array}{c}8(19.5) \\
25 \\
(61.0) \\
8(19.5) \\
\end{array}$ & $\begin{array}{c}8(19.5) \\
26(63.4) \\
7(17.1) \\
\end{array}$ & 0.931 \\
\hline $\begin{array}{l}\text { e) Ansiedad / Depresión } \\
\text { No tengo problemas } \\
\text { Tengo moderada } \\
\text { ansiedad/depresión }\end{array}$ & $\begin{array}{c}16 \\
(45.7) \\
16 \\
(45.7)\end{array}$ & $\begin{array}{l}14(40.0) \\
16(45.7)\end{array}$ & 0.550 & $\begin{array}{c}12 \\
(29.3) \\
24 \\
(58.5)\end{array}$ & $\begin{array}{l}13(31.7) \\
23(56.1)\end{array}$ & 0.946 \\
\hline Tengo mucha ansiedad/depresión & $3(8.6)$ & $5(14.3)$ & & $5(12.2)$ & $5(12.2)$ & \\
\hline
\end{tabular}

La intervención implementada se asoció a un descenso del dolor durante actividades básicas de la vida diaria (ABVD) $(p=0.0005)$, en el dolor durante los cuidados $(p=0.0005)$ y en el dolor durante el descanso $(p=0.001)$. Se apreció un mayor número de cuidadores en el grupo control con calidad de vida $>50$ puntos $(p=0.002)$ y con Índice de esfuerzo del cuidador $\leq 7$ puntos $(p=0.0005)$ respecto al grupo de intervención. El Índice de esfuerzo (Caregiver Burden Scale) descendió en ambos grupos; y aunque el porcentaje de cuidadores con puntuación $>7$ puntos fue mayor en el grupo de intervención que en el grupo control, la diferencia basal-final fue más 
relevante en el grupo de intervención que en el grupo control (-8.6\% vs $-2.5 \%)$ (tabla $5)$.

Tabla 5. Comparativa entre la situación basal y final (18 meses) de las variables resultado entre los grupos de estudio.

\begin{tabular}{|c|c|c|c|c|c|c|c|}
\hline \multirow{2}{*}{$\begin{array}{c}\text { Variables resultado, } \mathrm{N} \\
(\%)\end{array}$} & \multicolumn{3}{|c|}{ Intervención } & \multicolumn{3}{|c|}{ Control } & \multirow[b]{2}{*}{ Valor $p$} \\
\hline & Basal & 18 meses & Dif & Basal & 18 meses & Dif & \\
\hline $\begin{array}{l}\text { Dolor durante las } \\
\text { ABVD }\end{array}$ & & & & & & & 0.0005 \\
\hline No & $30(83,3)$ & $31(86,1)$ & 2.8 & $33(80,5)$ & $33(80,5)$ & 0.0 & \\
\hline $\begin{array}{l}\text { Sí } \\
\text { Dolor durante los }\end{array}$ & $6(16,7)$ & $5(13,9)$ & -2.8 & $8(19,5)$ & $8(19,5)$ & 0.0 & \\
\hline cuidados & & & & & & & 0.0005 \\
\hline No & $27(75,0)$ & $29(80,6)$ & 5.6 & $30(73,2)$ & $30(73,2)$ & 0.0 & \\
\hline $\begin{array}{l}\text { Sí } \\
\text { Dolor durante el }\end{array}$ & $9(25,0)$ & $7(19,4)$ & -5.6 & $11(26,8)$ & $11(26,8)$ & 0.0 & \\
\hline descanso & & & & & & & 0.001 \\
\hline No & $32(88,9)$ & $31(86,1)$ & -2.8 & $37(90,2)$ & $35(85,4)$ & -4.8 & \\
\hline Sí & $4(11,1)$ & $5(13,9)$ & 2.8 & $4(9,8)$ & $61(14,6)$ & 4.8 & \\
\hline Calidad de vida EQ-5D & & & & & & & 0.002 \\
\hline$<50$ & $5(15,6)$ & $6(18,8)$ & 3.2 & $6(15,8)$ & $6(15,8)$ & 2.8 & \\
\hline $\begin{array}{l}\quad \geq 50 \\
\text { Índice de esfuerzo del }\end{array}$ & $27(84,4)$ & $26(81,3)$ & -3.1 & $32(84,2)$ & $32(84,2)$ & -2.6 & \\
\hline cuidador & & & & & & & 0.0005 \\
\hline No & $12(34,3)$ & $15(42,9)$ & 8.6 & $19(46,3)$ & $20(48,8)$ & 2.5 & \\
\hline Sí & $23(65,7)$ & $20(57,1)$ & -8.6 & $22(53,7)$ & $21(51,2)$ & -2.5 & \\
\hline
\end{tabular}

ABVD: actividades básicas de la vida diaria; Dif: diferencia entre la situación final y basal. EQ-5D: cuestionario EuroQoL-5D de calidad de vida

El modelo multivariante mediante regresión logística binaria puso de manifiesto que controlando por las variables sociodemográficas de cuidadores informales y el nivel de conocimientos previos, tener un Índice de esfuerzo del cuidador basal alto (>7 puntos) incrementa 8.5 veces la probabilidad de tener un Índice de esfuerzo del cuidador final alto $(O R=8.5 ; I C 95 \%=1.7-42.3)(p=0.009)$, aunque no se detectaron diferencias con significación estadística $(p=0.606)$.

Para comprender mejor cómo influía el grado de discapacidad de la persona dependiente en el Índice de esfuerzo del cuidador agrupamos a los cuidadores según dicho índice de esfuerzo a lo largo del estudio y los comparamos con el índice de Barthel de la persona dependiente. Así, se crearon los siguientes grupos: grupo "a" reunía a cuidadores informales que puntuaron bajo en el Índice de esfuerzo del cuidador tanto en la medición basal como en la final (bajo/bajo); grupo "b" (alto/bajo); grupo "c" (bajo/alto) y grupo "d" (alto/alto). Se encontraron diferencias estadísticamente significativas y clínicamente relevantes en el porcentaje de pacientes que puntuaban alto a lo largo del estudio (grupo d) siendo menor en el grupo de intervención que en el grupo control (33.3\% vs 47.8\%) ( $p=0.035$ ) (tabla 6). Cuando se analizaron las puntuaciones del índice de Barthel en cada uno de estos grupos de Índice de esfuerzo del cuidador, se encontró que éste era peor en los cuidadores del grupo d, es decir, aquellos que mantuvieron un Índice de esfuerzo $>7$ a lo largo del estudio. Además, los cuidadores del grupo de intervención soportaron una 
carga mayor, con índice de Barthel más bajos, a medida que avanzaba el estudio $(p=0.035)$ que los del grupo control.

Tabla 6. Variación en la puntuación del índice de esfuerzo del cuidador (IEC) a lo largo del estudio según el grupo de estudio y la puntuación en el índice de Barthel (IB) de la persona dependiente expresado en media (DS) y su IC95\%.

IEC Final

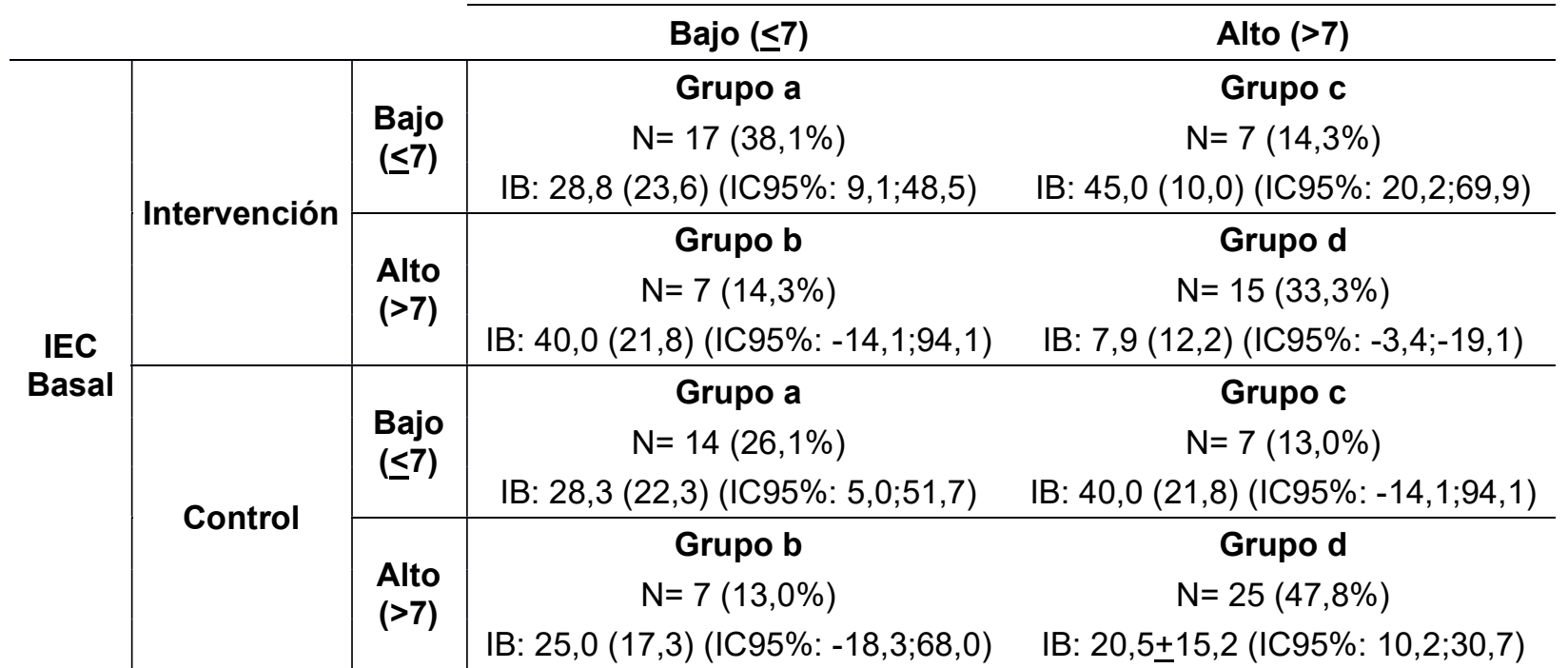

grupo "a": cuidadores informales que puntuaron bajo en el Índice de esfuerzo del cuidador tanto en la medición basal como en la final (bajo/bajo); grupo "b": alto/bajo; grupo "c": bajo/alto y grupo "d": alto/alto

\section{DISCUSIÓN}

Esta intervención educativa ha permitido una disminución estadísticamente significativa en la proporción de cuidadores familiares con dolor asociado a las actividades básicas de la vida diaria, a las tareas de cuidados y al descanso; mayor en el grupo de intervención que en el grupo control. Aunque la calidad de vida empeoró en ambos grupos, la proporción de personas que puntuaron menos de 5 puntos en la ENV del cuestionario EQ-5D fue mayor en el grupo de intervención que en el grupo control. En el mismo sentido, el Índice de esfuerzo del cuidador (Caregiver Burden Scale) descendió en ambos grupos; aunque el descenso en porcentaje de cuidadores con puntuación mayor de 7 puntos fue mayor en el grupo de intervención que en el grupo control.

Los cuidadores informales han sido familiares de las personas dependientes en más del $80 \%$ de los $\operatorname{casos}^{(11)}$. Este rol de cuidador ha recaído fundamentalmente en la mujer, dato que se repite en otros estudios sobre el cuidado informal de personas dependientes, recayendo principalmente sobre la esposa y/o la hija ${ }^{(20,21)}$, con una edad media entre 55 y 65 años y con un nivel de estudios medio ${ }^{(22,23)}$.

Los resultados del presente estudio evidencian la vulnerabilidad de los cuidadores familiares a padecer problemas físicos y psíquicos, lo que algunos autores han definido como el "síndrome del cuidador»(22). El cuidado de personas dependientes ha sido identificado como uno de los eventos más estresantes del ciclo familiar ${ }^{(24)}$. Los cuidadores realizan diariamente una gran cantidad de tareas que repercuten negativamente en su salud física y emocional, siendo propensos a padecer 
determinadas patologías tales como el dolor de espalda, los problemas osteoarticulares y/o músculo-esqueléticos ${ }^{(25)}$ y problemas psicológicos como son la ansiedad y/o la depresión(26). Como indican Toribio-Díaz et al, el $36,4 \%$ de los cuidadores se mantienen laboralmente activos mientras que el $13,1 \%$ reducen la jornada laboral y un 5,4\% llega a abandonar su puesto de trabajo para atender adecuadamente a la persona dependiente ${ }^{(3)}$. Todos estos factores contribuyen a incrementar el Índice de esfuerzo del cuidador que se correlaciona con el dolor percibido por estos cuidadores ${ }^{(27)}$.

Ortiz, en su tesis doctoral publicada en $2019^{(26)}$, concluye que el perfil basal de los cuidadores informales es el de una persona con sobrecarga intensa, dolor lumbar e incapacidad moderada; que además perciben un escaso apoyo social y una limitada calidad de vida. En nuestro estudio, dichos cuidadores presentan en el estado basal un perfil similar al descrito por este autor.

Según los autores analizados, en relación al cuestionario EuroQoL-5D, los cuidadores familiares de enfermos de Alzheimer presentaban más problemas en las dimensiones de movilidad, actividades cotidianas, dolor o malestar y ansiedad o depresión que la población general, tras ajustar por edad y sexo. Esto también se refleja en la valoración que asignan al estado de salud general (ENV) del EuroQoL-5D, en la que la muestra de cuidadores obtuvo también una peor valoración en su calidad de vida que la población general ${ }^{(22,28)}$.

A medida que va pasando el tiempo, el porcentaje de cuidadores informales con Índice de esfuerzo del cuidador alto va disminuyendo en ambos grupos, lo que puede explicarse mediante la "hipótesis de la adaptación", en la que se plantea que el cuidador, a pesar del progresivo deterioro de la persona dependiente, aprende a adaptarse a la situación, pudiendo experimentar una mejoría. Según esta hipótesis la fase inicial de los cuidados a una persona dependiente sería el peor momento para los cuidadores ${ }^{(23,29,30)}$. En nuestro estudio, este descenso fue más notable en el grupo de intervención que en el grupo control, lo cual puede deberse a la efectividad de la intervención multidimensional.

El Índice de esfuerzo del cuidador (Caregiver Burden Scale $>7$ puntos) se asocia a una peor calidad de vida y mayor nivel del dolor percibido (ENV) lo que concuerda con los resultados del estudio de Gómez-Pisano et al ${ }^{(31)}$.

Nuestra intervención consistió en un programa multidimensional en el que se abordaban medidas de cinesiterapia, control postural y ergonomía de los cuidados y movilizaciones y técnicas de relajación. Existen experiencias previas que apoyan la eficacia de las intervenciones educativas en personas cuidadoras. La bibliografía consultada muestra que los programas han sido de corta duración y han estado enfocados a alguna modalidad concreta de intervención: a promover la actividad física, la higiene postural, la ergonomía, etc.; solo algunos de ellos abordan múltiples modalidades de intervención, a corto-medio plazo. A pesar de ello, concluyen que mejoran el estado de salud de la persona cuidadora. De esta manera, el ensayo clínico aleatorizado realizado por Araujo sobre 33 cuidadores concluyó que una intervención de 12 semanas de duración con entrenamiento en higiene postural, combinado con cinesiterapia y adaptaciones en el hogar pueden reducir el dolor y mejorar varios aspectos de la calidad de vida en cuidadores familiares ${ }^{(10)}$. En esta misma línea encontramos el estudio de Bezerra et al, que recomiendan la adopción 
de medidas ergonómicas simples, posturas adecuadas de trabajo, y la realización de ejercicios de preparación para el trabajo diario (ejercicios de reeducación postural global y ejercicios de estiramiento muscular tanto analítico como global), para minimizar los factores de riesgo de lesiones, disminuir el dolor lumbar y mejorar la calidad de vida ${ }^{(32)}$. Coincidiendo con estos y otros autores, se puede decir que el establecimiento de hábitos saludables en el día a día de los cuidadores puede mejorar su calidad de vida, disminuir el estrés y frenar el deterioro de su salud. Por contra, siguiendo las conclusiones de Larrañaga ${ }^{(33)}$, la menor dedicación de las personas cuidadoras a la actividad física y al descanso puede explicar que tengan peor respuesta en las puntuaciones en salud mental, y en nuestro caso en el dolor y calidad de vida.

La subjetividad de las respuestas, especialmente en lo referido al dolor y calidad de vida, ha podido asociarse a un sesgo de información. El requerimiento de tiempo percibido por los cuidadores informales para ejercer esta tarea, les limita la asistencia a los talleres e incluso puede ser causa de renuncia para participar en el proyecto.

El diseño de nuestro estudio limitó esta actividad a los cuidadores familiares principales, no teniendo en cuenta los casos en los que la responsabilidad de los cuidados depende de varios cuidadores que rotan en esta función. De hecho, GómezRamos y González-Valverde valoran este papel en las personas cuidadoras (47\%) aunque no hacen un análisis específico de su impacto en las variables que estudiaron ${ }^{(21)}$.

Por todo ello, podemos decir que se hace necesaria la implementación de actividades multidisciplinares de prevención y de promoción de la salud dirigidas a apoyar a los cuidadores informales de personas dependientes, ya que la literatura existente avala los beneficios asociados a las mismas tanto para la persona atendida como para los cuidadores, disminuyendo la sobrecarga y el dolor percibido por éstos y los ingresos hospitalarios de las personas dependientes ${ }^{(34-36)}$.

Según los resultados de nuestro estudio y otros trabajos publicados relevantes en esta materia, la implementación de actividades dirigidas a la formación multidimensional, disminuyen los síntomas de dolor, disminuye el esfuerzo del cuidador y mejora la calidad de vida. Esto puede ser relevante para la disminución del coste de estas enfermedades musculoesqueléticas y osteoarticulares.

Para ello, sería recomendable diseñar estudios longitudinales con seguimientos intermedios para mejorar la adherencia del cuidador al programa y determinar el coste/efectividad de estas intervenciones para su posterior implementación en los cuidadores informales.

\section{CONCLUSIONES}

Las conclusiones obtenidas sobre el estudio que hemos realizado sobre cuidadores informales de nuestro ámbito sanitario nos indican que los programas de intervención en cuidadores informales de pacientes dependientes resultan efectivos para mejorar el estado de salud y la calidad de vida de este colectivo por lo que se debe fomentar su realización desde los servicios de Atención Primaria. 


\section{REFERENCIAS}

1. World Health Organization (WHO). Envejecimiento y salud [Internet]. World Health Organization. Ginebra, Suiza: WHO Press, World Health Organization; 2018 [cited 2018 Mar 12]. Available from: https://www.who.int/es/newsroom/fact-sheets/detail/envejecimiento-y-salud

2. Pimouguet C, Lavaud T, Dartigues JF, Helmer C. Dementia case management effectiveness on health care costs and resource utilization: a systematic review of randomized controlled trials. J Nutr Health Aging [Internet]. 2010 Oct;14(8):669-76. Available https://link.springer.com/content/pdf/10.1007/s12603-010-0314-4.pdf

3. Toribio-Díaz ME, Medrano-Martínez V, Moltó-Jordá JM, Beltrán-Blasco I. Red de cuidadores informales de los pacientes con demencia en la provincia de Alicante, descripción de sus características. Neurologia [Internet]. 2013;28(2):95-102. Available from: http://dx.doi.org/10.1016/j.nrl.2012.03.010

4. Garcia-Calvente M del M, Mateo-Rodriguez I, Maroto-Navarro G. El impacto de cuidar en la salud y la calidad de vida de las mujeres. Gac Sanit [Internet]. 2004;18 Suppl 2:83-92. Available from: https://doi.org/10.1157/13061998

5. Bocca Peralta G, Iglesias B A, Maridueña Arciniegas V. Dolor lumbar inespecífico en cuidadores de niños con discpacidad. Rev Mex Med Fis Rehab [Internet]. 2017;29(3-4):42-5. Available from: www.medigraphic.org.mx

6. Seira Lledós MP, Aller Blanco A, Calvo Gascón A. Morbilidad sentida y diagnosticada en cuidadores de pacientes inmovilizados de una zona de salud rural. Rev Esp Salud Publica [Internet]. 2002;76(6):713-21. Available from: https://www.mscbs.gob.es/biblioPublic/publicaciones/recursos_propios/resp/revi sta_cdrom/vol76/vol76_6/RS766C_713.pdf

7. Zuluaga Viscaya KA, Estrada Muñoz J. Evaluación del riesgo de patologías lumbares en cuidadores familiares de pacientes hospitalizados en casa con algún grado de dependencia por alteración de la movilidad utilizando el método MAPO. Rev Ing Ind [Internet]. 2019;6(6):9-16. Available from: https://repository.upb.edu.co/bitstream/handle/20.500.11912/6595/Evaluación del riesgo de patologías. pdf?sequence=1\&isAllowed=y

8. Yalcinkaya EY, Önes K, Ayna AB, Turkyilmaz AK, Erden N. Low back pain prevalence and characteristics in caregivers of stroke patients: A pilot study. Top Stroke Rehabil [Internet]. 2010;17(5):389-93. Available from: https://www.tandfonline.com/doi/abs/10.1310/tsr1705-389

9. Bardak AN, Erhan B, Gündüz B. Low back pain among caregivers of spinal cord injured patients. J Rehabil Med [Internet]. 2012;44(10):858-61. Available from: https://www.medicaljournals.se/jrm/content/abstract/10.2340/16501977-1043

10. de Araujo Freitas Moreira KL, Abalos-Medina GM, Villaverde-Gutierrez C, Gomes de Lucena NM, Belmont Correia de Oliveira A, Perez-Marmol JM. Effectiveness of two home ergonomic programs in reducing pain and enhancing quality of life in informal caregivers of post-stroke patients: A pilot randomized controlled clinical trial. Disabil Health J [Internet]. 2018 Jul;11(3):471-7. Available from: https://www.sciencedirect.com/science/article/abs/pii/S1936657418300049?via \%3Dihub

11. Downie WW, Leatham PA, Rhind VM, Wright V, Branco JA, Anderson JA. Studies with pain rating scales. Ann Rheum Dis. 1978;37(4):378-81.

12. Lázaro C, Torrubia R, Baños JE. El cuestionario de dolor de McGill. Dolor 
Investig Clínica Ter [Internet]. 2007;22(2):69-77. Available from: http://www.dolor.es

13. Lázaro C, Caseras X, Whizar-Lugo VM, Wenk R, Baldioceda F, Bernal R, et al. Psychometric properties of a Spanish version of the Mcgill Pain Questionnaire in several Spanish-speaking countries. Clin J Pain. 2001;17(4):365-74.

14. López AS, Moral SM. Validación del Indice de Esfuerzo del Cuidador en la población española [Validation of the Caregiver Strain Index in Spanish population]. Enferm Comunitaria. 2005;1(May):12-7.

15. Herdman M, Badia X, Berra S. El EuroQol-5D: una alternativa sencilla para la medición de la calidad de vida relacionada con la salud en atención primaria. Atención Primaria. 2001;28(6):425-9.

16. Badia X, Roset M, Montserrat S, Herdman M, Segura A. La version espanola del Euro-QoL : descripcion y aplicaciones. Med Clin (Barc). 1999;112 Suppl:79-85.

17. Rodríguez Chamorro MÁ, García-Jiménez E, Amariles $\mathrm{P}$, Rodríguez Chamorro A, José Faus M. Revisión de tests de medición del cumplimiento terapéutico utilizados en la práctica clínica. Aten Primaria. 2008;40(8):413-7.

18. Morisky DE, Green LW, Levine DM. Concurrent and predictive validity of a selfreported measure of medication adherence. Med Care. 1986 Jan;24(1):67-74.

19. Piñeiro I, Rodríguez S, Albite A, Freire C, Ferradás M del M. Sobrecarga y salud percibida en cuidadores informales de pacientes con enfermedad mental. Eur $\mathrm{J}$ Heal Res [Internet]. 2017 Dec 12;3(3):185-96. Available from: https://formacionasunivep.com/ejhr/index.php/journal/article/view/75

20. Ortiz-Mallasen V, Trullenque-Vicario J, Claramonte-Gual E. Dolor de espalda en cuidadores no profesionales de personas adultas dependientes: Estudio descriptivo. ICUE Investig y Cuid Enfermería [Internet]. 2016 Jan 11;1(3):12. Available from: http://www.revistaicue.es/revista/ojs/index.php/ICUE/article/view/34

21. Gómez-Ramos M, González-Valverde F. El cuidador del paciente con demencia: aplicación del test Índice del Esfuerzo del Cuidador. Rev Esp Geriatr Gerontol [Internet]. 2004;39(3):154-9. Available from: https://www.sciencedirect.com/science/article/pii/S0211139X04749495?casa_to ken=fNg2k09I6YsAAAAA:X8OHq3xS2h-

6ABZ01s2g8sHx7T7N7M6MJDYkiwne0hkYSZZwcXRD0hOdM57gcDvxUdHdlH PO5A

22. Crespo López M, López Martínez J. El estrés en cuidadores de mayores dependientes. Cuidarse para cuidar. Madrid, España: Pirámide Ediciones; 2007. $280 \mathrm{p}$.

23. Badia Llach X, Lara Suriñach N, Roset Gamisans M. Calidad de vida, tiempo de dedicación y carga percibida por el cuidador principal informal del enfermo de Alzheimer. Atención Primaria [Internet]. 2004;34(4):170-7. Available from: https://www.ncbi.nlm.nih.gov/pmc/articles/PMC7668642/pdf/main.pdf

24. Zarit SH, Reever KE, Bach-Peterson J. Relatives of the impaired elderly: correlates of feelings of burden. Gerontologist [Internet]. 1980 Dec;20(6):64955. Available from: https://pubmed.ncbi.nlm.nih.gov/7203086

25. Hernández Rojas EM, Murillo Álvarez S, Solano Bastos A, Enríquez-Reyna MC. Riesgo de alteraciones músculo-esqueléticas en cuidadores informales de personas con parálisis cerebral. Rev Psicol del Deport [Internet]. 2017;26(2):107-12. Available from: https://www.redalyc.org/pdf/2351/235152045015.pdf

26. Ortiz Mallasen V. Estudio experimental sobre la eficacia de una intervención educativa individual en el dolor lumbar de cuidadores no profesionales de 
personas dependientes. Programa TRANSFE [Internet]. [Castelló de la Plana]: Universitat Jaume I; 2019 [cited 2020 Sep 17]. Available from: http://hdl.handle.net/10803/667826

27. Villarejo Aguilar L, Zamora Peña M, Casado Ponce G. Sobrecarga y dolor percibido en cuidadoras de ancianos dependientes. Enferm Glob [Internet]. 2012;27:159-65. Available

from: https://revistas.um.es/eglobal/article/view/eglobal.11.3.155111/136241

28. Casal Rodríguez B, Rivera Castiñeira B, Currais Nunes L. [Alzheimer's disease and the quality of life of the informal caregiver]. Rev Esp Geriatr Gerontol [Internet]. 2019;54(2):81-7. Available from: https://www.elsevier.es/es-revistarevista-espanola-geriatria-gerontologia-124-pdf-S0211139X18306905

29. Losada-Baltar A, Peñacoba-Puente C, Marquez-Gonzalez M, Cigaran-Mendez M. Cuidar cuidándose, evaluación e intervención interdisciplinar con cuidadores familiares de personas con demencia. Madrid, España Encuentro, Caja Madrid. 2008;

30. Manso Martínez ME, Sánchez López M del P, Cuéllar Flores I. Salud y sobrecarga percibida en personas cuidadoras familiares de una zona rural. Clin y Salud [Internet]. 2013;24(1):37-45. Available from: https://reader.elsevier.com/reader/sd/pii/S1130527413700057?token=7DF5FEC 3D4333BDF2DC12AAD0FB80686DDA6F95DD0F48D1DA28B871F324C9261D 20856175FF0D62B50F1BE1494DA3592\&originRegion=eu-west-

1\&originCreation $=20210427102832$

31. González-Pisano AC, Granado-Villacé R, García-Jáñez E, del Cano-González $\mathrm{C}$, Fernández-Fernández Ml. Calidad de vida relacionada con la salud en cuidadoras de personas dependientes de dos zonas rurales de León. Enferm Clin [Internet]. 2009;19(5):249-57. Available from: https://www.elsevier.es/esrevista-enfermeria-clinica-35-linkresolver-calidad-vida-relacionada-con-saludS1130862109001351

32. Bezerra Ribeiro S, Gatto Cárdia MC, Almeida LC. Biomechanical and organizational risk and prevalence of low back pain in the old adults caregivers of a nursing home in Joao Pessoa/PB. Work [Internet]. 2012;41(Suppl.1):19339. Available from: Biomechanical and organizational risk and prevalence of low back pain in the old adults caregivers of a nursing home

33. Larrañaga I, Martín U, Bacigalupe A, María Begiristáin J, José Valderrama M, Arregi B. Impacto del cuidado informal en la salud y la calidad de vida de las personas cuidadoras: análisis de las desigualdades de género. Gac Sanit [Internet]. $2008 ; 22(5): 443-50 . \quad$ Available from: http://dx.doi.org/10.1157/13126925

34. López J, Crespo M. Intervenciones con cuidadores de familiares mayores dependientes: Una revisión. = Interventions for caregivers of older and dependent adults: A review. Psicothema [Internet]. 2007;19(1):72-80. Available from:

http://ezproxy.msvu.ca/login?url=http://search.ebscohost.com/login.aspx?direct= true \&db=psyh\&AN=2007-01207-011\&site=ehost-

live\&scope=site\%0Ahttp://mcrespo@psi.ucm.es

35. Moreno Rodríguez R, Cigarán M, Peñacoba Puente C, Montorio Cerrato IM, Losada Baltar A. Análisis de programas de intervención psicosocial en cuidadores de pacientes con demencia. Inf psiquiátricas [Internet]. 2006;(184):173-86. Available from: http://www.informacionespsiquiatricas.com/anteriores/info_2006/02_184_08.htm

36. Torres Egea MP, Ballesteros Pérez E, Sánchez Castillo PD. Programas e 
intervenciones de apoyo a los cuidadores informales en España. Gerokomos [Internet]. 2008;19(1):9-15.

Available

from:

http://scielo.isciii.es/pdf/geroko/v19n1/rincon1.pdf 